\title{
O REFLEXO CUTÂNEO-PLANTAR EM EXTENSÃO
}

\author{
(BABINSKI, 1896/1898)
}

\section{R. OLIVEIRA-SOUZA* W. MARTIGNONI DE FIGUEIREDO**}

\begin{abstract}
RESUMO - A dois anos do centenário da identificação do reflexo cutâneo-plantar em extensão como sinal de afecção do sistema nervoso central, pareceu-nos oportuno traduzir passagens relevantes de Babinski sobre o fenômeno que descreveu. Há indícios de que um dos motos mais influentes na criação do espírito de época que facilitou a valorização clínica do "fenômeno dos artelhos", deveu-se à exigência pragmática de se distinguir paralisias "histéricas" de "orgânicas" para aplicação médico-legal imediata.
\end{abstract}

PALAVRAS-CHAVE: Babinski, hemiplegia, reflexos, sistema piramidal.

\section{The extensor plantar reflex (Babinski, 1896/1898)}

SUMMARY - The extensor plantar reflex was described by Babinski in 1896. Given the obvious relevance of the sign for internal medicine as well as the paucity of translations of the original sources into Portuguese, we thought it timely to recall the ingenious arguments Babinski used to demonstrate his views on the "toe phenomenon", as he would call it. A careful analysis of Babinski's writings suggests, further, that he was driven by keen intuition as well as by medico-legal interests.

KEY WORDS: Babinski, hemiplegia, reflexes, pyramidal system.

A produção abundante e cada vez mais especializada do conhecimento médico frequentemente dificulta o acesso dos mais jovens às nascentes da revolução diagnóstica e terapêutica que ora vivemos. A lucidez da descrição original de Babinski, a dois anos de seu centenário, incentivou-nos a traduzila na íntegra. Ao abordar um dos sinais mais conhecidos da semiologia médica, esperamos reanimar o cultivo de clássicos, que, diariamente, reencenamos à beira do leito.

\section{O TEXTO'}

Sur le réflexe cutané plantaire dans certaines affections organiques du système nerveux central J'ai observé dans un certain nombre de cas d'hémiplégie ou de monoplégie crurale liée à une affection organique du système nerveux central une perturbation dans le réflexe cutané plantaire donc voici en quelques mots la description. $\mathrm{Du}$ côte sain la piqûre de la plante du pied provoque,
Sobre o reflexo cutâneo-plantar em determinadas afecções orgânicas do sistema nervoso central

Tenho observado, em alguns casos de hemiplegia ou de monoplegia crural ligadas a uma afecção orgânica do sistema nervoso central, uma perturbação do reflexo cutâneoplantar, cuja descrição aqui está, em poucas palavras. Do lado são, a picada da planta do

"Professor Assistente, Serviço de Clínica Médica C (Prof. Omar da Rosa Santos), Hospital Universitário Gaffrée e Guinle, Universidade do Rio de Janeiro (HUGG - UNI-RIO); "'Professor Adjunto, Serviço de Clínica Médica C (Prof. Omar da Rosa Santos), HUGG - UNI-RIO. Aceite: 1-outubro-1994.

Dr. Ricardo de Oliveira Souza - Rua General Belford 226 - 20961-000 Rio de Janeiro RJ - Brasil. 
comme cela a lieu d'habitude à l'état normal, une flexion de la cuisse sur le bassin, de la jambe sur la cuisse, du pied sur la jambe et des orteils sur le métatarse. Du côté paralysé, une exciation sembable donne lieu aussi à une flexion de la cuisse sur le bassin, de la jambe sur la cuisse et du pied sur la jambe, mais les orteils, au lieu de se fléchir, exécutent un mouvement d'extension sur le métatarse.

II m'a été donné d'observer ce trouble dans des cas d'hémiplégie récente remontant à quelques jours seulement, ainsi que dans des cas d' hémiplégie spasmodique de plusiers mois de durée; je l'ai constaté chez des malades qui étaient incapables de mouvoir volontairement les orteils, comme aussi sur des sujets qui pouvaient encore faire éxecuter aux orteils des mouvements volontaires; mais je dois ajouter que ce trouble n'est pas constant.

J'ai aussi observé dans plusiers cas de paraplégie crurale due à une lésion organique de la moelle un mouvement d'extension des orteils à la suite de la piqûre de la plante du pied, mais, comme en pareil cas, il n'y a pas chez malade même de point de comparison, la réalité d'un trouble est moins manifeste.

En résumé, le mouvement réflexe consécutif à la piqûre de la plante du pied peut subir dans les paralysies crurales reconnaissant pour cause une affection organique du système nerveux central non seulement, comme on le sait, une modification dans son intensité, mais aussi une perturbation dans sa forme. pé provoca, como habitualmente sucede no estado normal, a flexão da coxa sobre a bacia, da perna sobre a coxa, do pé sobre a perna e dos artelhos sobre o metatarso. Do lado paralisado, excitação semelhante dá lugar, também, à flexão da coxa sobre a bacia, da perna sobre a coxa $\mathrm{e}$ do pé sobre a perna, mas os artelhos, ao invés de se fletirem, executam um movimento de extensão sobre o metatarso.

Pude verificar este distúrbio em casos de hemiplegia recente, remontando a apenas alguns dias, bem como em casos de hemiplegia espamódica ${ }^{\mathrm{a}}$ com vários meses de duração; constatei-o em doentes impossibilitados de mover voluntariamente os artelhos, como, também, em indivíduos que ainda podiam fazer os artelhos executar movimentos voluntários; devo, porém, acrescentar que tal distúrbio não é constante.

Tenho igualmente observado em diversos casos de paraplegia crural devida a uma lesão orgânica da medula, um movimento de extensão dos artelhos que se segue à picada da planta do pé, mas, como em tais casos nāo há qualquer ponto de comparação no mesmo doente, a realidade de algum distúrbio pode não ser tão evidente.

Em resumo, o movimento reflexo consecutivo à picada da planta do pé pode sofrer, nas paralisias crurais reconhecendo por causa uma afecção orgânica do sistema nervoso central, não somente, como se sabe, uma modificação em sua intensidade, mas, também, uma perturbação na sua forma.

a"Espasmódico" e "espástico" constituem exemplos de galicismo e anglicismo incorporados ao nosso léxico técnico em épocas distintas do conhecimento médico. "Espasmódico" ocorreu na literatura até, aproximadamente, a II Guerra Mundial. Aloysio de Castro, em sua Semiotica ${ }^{8}$, escreveu: "Expoente máximo da hipertonia muscular, é na condiçāo de rigidez espamódica e prolongada (...), que está o fundamento da contratura" (em itálico no original). "Spastic", todavia, aparecia nos textos ingleses do século XIX para descrever os mesmos fenômenos clínicos - por exemplo, em 1893 Gowers já se referia à "hemiplegia espática"13 e à "paraplegia espática"12 -, mas apenas depois da II Guerra assumiu a dianteira na linguagem corrente. Provavelmente, se referiam não apenas ao fenômeno espático das lesões piramidais - conforme hoje o concebemos - mas, também, às formas fixas de distonia.

\section{O PROBLEMA}

Joseph François Félix Babinski tinha 32 anos quando expôs suas observações preliminares para a Sociedade de Biologia de Paris, em 22 de fevereiro de $1896^{\prime}$ (Figura). A ênfase no fator "orgânico" permeia o texto e vinculou-se diretamente à oportunidade da descoberta, pois, como ele 


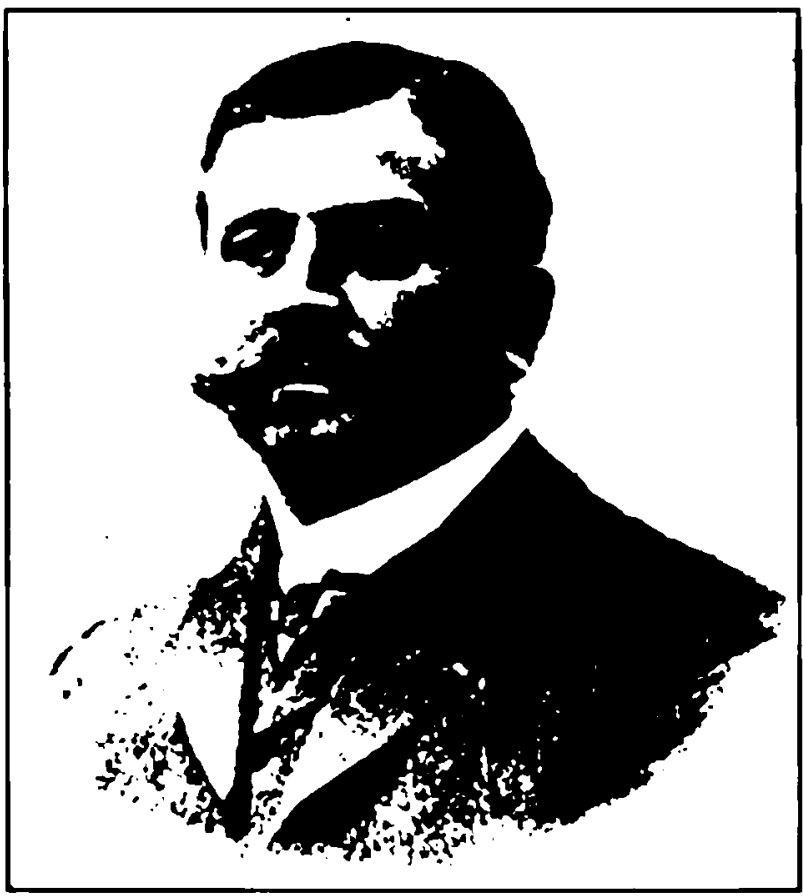

Babinski (1857-1932) aos 47 anos (Fotografia de autor desconhecido, gentilmente cedida por J. van Gijn, que a obteve da Biblioteca Médica e Farmacêutica, em Haia).

próprio teria declarado a Wartenberg, muitos neurologistas na época viviam ocupados em encontrar meios de diagnóstico diferencial entre as hemiplegias "histéricas" e "orgânicas"23. Babinski acreditou que o "fenômeno dos artelhos" - como se referia à resposta extensora - solucionaria o problema. Nas "hemiplegias histéricas":

\section{(...) le mouvement reflexe consecutif à l'excitation} de la plante du pied ne subit pas d'inversion dans sa forme. Le phénomène des orteils fait défault.
(...) o movimento reflexo consecutivo à excitação da planta do pé não sofre inversão em sua forma. O fenômeno dos artelhos está ausente.

O conjunto da obra de Babinski deixa claro que a distinção entre "histéricos" e "orgânicos" não se restringia a mero formalismo teórico ou acadêmico. Na realidade, seu maior interesse parecia centrar-se nos aspectos médico-legais de determinados casos individuais, que pudessem auxiliá-lo na tomada de decisões em consultorias técnicas ${ }^{5,7}$.

\section{TÉCNICA, ESTIMULO E RESPOSTA}

O método originalmente empregado para suscitar o reflexo consistia na picada da planta do pé. Babinski não disse como procedia: se, por exemplo, aplicava o estímulo (estilete ou agulha ${ }^{6}$ ) de maneira rápida e repetida (alfinetadas), ou sob pressão graduada e contínua contra a pele. A determinação da região do pé mais eficaz para a produção da resposta, assim como a definição do melhor modo de estimulaçāo' - que até hoje utilizamos e que se seguiu à estimulação por picadas -, seriam definidos pouco depois, no relatório definitivo, em que restringiu o valor de sinal à extensão do hálux ${ }^{3}$. Este trabalho havia sido precedido de breve discussão no Congresso Internacional de Neurologia, Psiquiatria, Eletricidade Médica e Hipnologia, realizado em Bruxelas em 1897². 
(...) A l'état normal, chez l'adulte, le chatouilement ou la piqûre de la plante du pied provoque ordinairement la flexion des orteils sur le métatarse et jamais l'extension. Or, dans certaines affections organiques du système nerveux central, une excitation sembable donne parfois lieu à une extension des orteils sur le metatarse. Dans certaines cas, les cinq orteils s'étendent; dans d'autres cas, l'extension ne porte pas que sur le gros orteil.
(...) No adulto normal, a estimulação da planta do pé por cócegas ou picada, provoca, ordinariamente, a flexão dos artelhos sobre o metatarso, jamais a extensão. Em determinadas afecções orgânicas do sistema nervoso central, excitação semelhante às vezes dá lugar à extensão dos artelhos sobre o metatarso. Em certos casos, os cinco se estendem; em outros, a extensão se assenta somente sobre o grande artelho.

\section{DA OBSERVAÇÃO AO SINAL ${ }^{15}$}

A rigor, a inversão do reflexo plantar havia sido notada antes de Babinski, mas não recebeu valor diagnóstico. Remak, entre outros, registrara a extensão do hálux em resposta à excitaçāo da planta do pé em um paciente com "mielite torácica transversa"21. Todavia, quando, ainda hoje, nos atemos à brusca retirada reflexa de uma perna paralisada à ação da vontade, compreendemos porque, por tanto tempo, se emprestou tão pouca atenção à fração digital da resposta. Despojando-se dessa atitude e concentrando atenção nos movimentos dos dedos, Babinski definiu as respostas flexora e indiferente como normais, ao mesmo tempo em que isolou a extensão dos artelhos como sinal. Inicialmente genérico, logo passou a interpretá-la como indicador de "afeç̧ão orgânica" do sistema nervoso central. A palavra "afecção", como hoje, designava qualquer processo mórbido abstraído de etiologia, frequentemente expresso pelo sufixo grego patia precedido do nome do órgão acometido ${ }^{16}$.

A correlação entre o sinal e, mais precisamente, lesões totais ou parciais do feixe piramidal, foi também por ele inferida, logo depois, com base na observação de pacientes com lesões cerebrais e medulares, bem como em recém-nascidos sadios ${ }^{2}$ :

(...) Chez le nouveau-né, à 1 'état normal, l'excitation de la plante du pied donne lieu à de l'extension des orteils. Ce fait est sourtout interessant si on le rapproche de cet outre fait, à savoir que chez l'adulte c'est particulièrement dans les affections qui atteignent le système pyramidal qu'on observe le phénomène des orteils.
(...) No recém-nascido normal, a excitação da planta do pé dá lugar à extensão dos artelhos. Tal fato é sobretudo interessante quando cotejado com este outro, a saber, que, no adulto, o fenômeno dos artelhos é particularmente observado nas afecções que atingem o sistema piramidal.

Um dos melhores estudos sobre a organização do reflexo plantar normal e patológico foi feito por Nö̈ca e Sakelaru ${ }^{18}$. Nele, discutem a anatomia segmentar do reflexo - dos campos receptores periféricos aos efetores neuromusculares -, passando pelas conexões medulares relacionadas. Mais recentemente, suas observações foram revistas e elaboradas por van Gijn".

\section{VALOR SEMIOLÓGICO}

No trabalho fundamental, Babinski adotou o estilo de "apresentaçāo de pacientes"3. Relatando 8 casos em formato de vinhetas, enfatizou os aspectos que melhor ilustravam seus argumentos. Os dados salientes sobre cada um estão sumariados no Quadro.

Discutindo suas próprias observações, Babinski enfatizou a estreita relação do sinal com a hemiplegia e a paraplegia de lesões encefálicas e medulares variadas, independente de duração e extensão anatômica. A seu ver, o valor semiológico do sinal se sobressaía nos casos em que o estado dos reflexos tendinosos impedia a avaliação da integridade do sistema piramidal3: 
Quadro. Apresentaçāo dos pacientes.

\begin{tabular}{|c|c|c|}
\hline Paciente & Idade/Sexo & Dados Clínicos \\
\hline 1 & */F & $\begin{array}{l}\text { Hemiplegia esquerda de } 3 \text { anos, associada a contratura, hiperreflexia tendinosa e } \\
\text { marcha ceifante, atribuída a lesão do hemisfério cerebral direito. Movimentos } \\
\text { voluntários dos artelhos quase abolidos do lado afetado, onde a estimulação plantar } \\
\text { dá lugar à extensão, mais intensa nos dois primeiros artelhos; à direita, estimulação } \\
\text { idêntica produz resposta em flexão. }\end{array}$ \\
\hline 2 & */F & $\begin{array}{l}\text { Hemiplegia esquerda de } 2 \text { anos, boa recuperação dos movimentos voluntários dos } \\
\text { artelhos. Plantar direito em flexāo; extensor à esquerda. }\end{array}$ \\
\hline 3 & $* / F$ & $\begin{array}{l}\text { Hemiplegia direita de } 1 \text { ano, seguida de hemiplegia contralateral } 1 \text { mês depois; "... a } \\
\text { extensão dos artelhos pode ser provocada dos dois lados, porém é mais pronunciada à } \\
\text { direita, onde a paralisia é maior". }\end{array}$ \\
\hline 4 & $* / F$ & $\begin{array}{l}\text { Paraplegia espástica, hiperreflexia e clônus do pé, por presumível placa de esclerose } \\
\text { ocupando a regiāo dorsal da medula lombar. Extensão extremamente intensa dos } \\
\text { cinco dedos, "ainda mais acentuada do que nos casos anteriores". }\end{array}$ \\
\hline 5 & $* / \mathbf{M}$ & $\begin{array}{l}\text { Mal de Pott. Paraplegia espástica, exagero dos reflexos tendinosos e impossibilidade } \\
\text { de ficar de pé. Extensão reflexa dos artelhos. Evoluiu com recuperação gradual da } \\
\text { capacidade de sustentação ortostática c de dar alguns passos. "A excitação da planta } \\
\text { do pé determina, agora, inicialmente, flexão dos artelhos, mas, se esta excitação é } \\
\text { repetida muitas vezes, os artelhos, a partir de determinado momento, passam a se } \\
\text { estender". }\end{array}$ \\
\hline 6 & $* / \mathrm{M}$ & Paraplegia espática de origem sifilítica; "fenômeno dos artelhos" presente. \\
\hline 7 & $* / \mathbf{M}$ & $\begin{array}{l}\text { Síndrome de Brown-Séquard (hemiparaplegia medular com anestesia cruzada), por } \\
\text { traumatismo raquiano por arma-de-fogo; à esquerda, paralisia espástica e extensão } \\
\text { dos artelhos; à direita, ligeira extensāo do hálux. }\end{array}$ \\
\hline 8 & $26 / \mathrm{F}$ & $\begin{array}{l}\text { Tabes dorsalis e meningoencefalite difusa. Atrofia óptica direita; sinal de } \\
\text { Argyll-Robertson, dores fulgurantes, arreflexia tendinosa, marcha quase normal. "A } \\
\text { extensão dos artelhos, neste caso, é das mais notáveis". }\end{array}$ \\
\hline
\end{tabular}

* Idade nāo reportada.

système pyramidal s'associe à une altération des racines postérieures, la présence du phénomène des orteils dans un cas de tables sera un indice important en révélant l'existence d'un trouble dans le système pyramidal, qui, sans ce signe, aurait pu être méconnu.

\section{COMENTÁRIOS}

Transcriçōes como as acima nos remetem diretamente à questão do papel da semiologia em um mundo de alta tecnologia. Se taticamente evitarmos as implicações filosóficas do tema, veremos que, a despeito da aguçada capacidade de classificação e descrição dos autores do passado, ainda há muito por fazer, em particular na determinação da especificidade, da sensibilidade, da confiabilidade e da validade de cada um dos principais sinais da semiologia tradicional. Por exemplo, Sawyer et al. constataram uma sensibilidade de $45 \%$, contra $100 \%$ de especificidade para o sinal de Babinski em uma série de 62 pacientes com lesões mono-hemisféricas ${ }^{22}$. Dos 20 pacientes com hemiplegia motora pura que publicamos há alguns anos, 16 tinham sinal de Babinski do lado oposto à lesão ${ }^{20}$. Em 100 casos de afecção cerebrovascular, Louis et al. verificaram que o exame neurológico seriado modificouse com o tempo, a frequência do sinal de Babinski correspondendo a $43 \%$ na primeira semana ${ }^{17}$. Tema de não menos importância é o do sinal em pessoas normais e em pacientes sem lesø̃es intracranianas estruturais: deixando de lado a conhecida resposta extensora dos recém-nascidos, $\varepsilon$ 
digna de nota a possibilidade de se evocar o sinal durante o sono ${ }^{10}$ e em determinados estados de inconsciência patológica ${ }^{14}$, bem como após longas caminhadas ${ }^{24}$, demonstrações da possibilidade de ocorrência do sinal por modificaçōes funcionais do sistema nervoso, na ausência de alteração estrutural.

O retorno sistemático às fontes deve ser incentivado, nem tanto pelo que possa significar como erudição, mas, sobretudo, por suas aplicaçōes práticas, vertente pouco valorizada em nossa literatura. A consulta dos textos antigos educa a percepção e instiga a imaginação, qualidades inerentes aos praticantes sensíveis. O potencial pedagógico desse procedimento não deve ser subestimado, face à semelhança entre a prática da neurologia contemporânea em muitas regiōes brasileiras e a que prevaleceu na Europa e nos Estados Unidos no final do século XIX ${ }^{19}$, quando os sentidos desarmados do clínico naturalmente se impunham ao instrumental acessório, ainda tão rudimentard.

Agradecimento - Agradecemos aos funcionários das bibliotecas do Instituto Oswaldo Cruz e do Centro de Ciências da Saúde da Universidade Federal do Rio de Janeiro pela diligência na localização dos textos originais, que permitiram a realização do presente estudo.

\section{REFERÊNCIAS}

1. Babinski J. Sur le réflexe cutané plantaire dans certaines affections organiques du système nerveux central. C R Soc Biol (Paris) 1896, 48: 207-208.

2. Babinski J. Valeur pathogénique et séméiologique des réflexes (discussion). Bull Méd 1897, 11: 896.

3. Babinski J. Du phénomène des orteils et de sa valeur sémiologique. Sem Méd 1898, 18: 321-322.

4. Babinski J. Diagnostique différentiel de l'hémiplégie organique et de l'hémiplégie hystérique (1900). Reproduzido em Oeuvre Scientifique. Paris: Masson 1934, p91-111.

5. Babinski J. De l'abduction de orteils. Rev Neurol (Paris) 1903, 11: 728-729.

6. Babinski J, Sur la transformation du régime des réflexes cutanés dans les affections du système pyramidal. Rev Neurol (Paris) 1904, 12: 58-62.

7. Babinski J. Introduction à la sémiologie des maladies du système nerveux: des symptômes objectifs que la volonté est incapable de reproduire.(De leur importance en médecine légale). Reproduzido em Oeuvre Scientifique. Paris: Masson 1934, p3-14.

8. Castro A. Semiotica nervosa. Ed 2. Rio de Janeiro: F. Briguiet \& Cia, 1935, p201.

9. Dohmmann GJ, Nowack WJ. The upgoing great toe: optimal method of elicitation. Lancet 1973, 1: 339-341.

10. Fujiki A, Shimizu A, Yamada Y, Yamamoto Y, Kaneko Z. The Babinski reflex during sleep and wakefulness. EEG Clin Neurophsyiol, 1971, 31:610-613.

11. Gijn J. The Babinski sign and the pyramidal syndrome. J Neurol Neurosurg Psychiatry 1978, 41: 865-873.

12. Gowers WR. A manual of diseases of the nervous system, Vol I: Diseases of nerves and spinal cord. Ed 2 (1893). Darien, Conn: Hafner Publ Co 1970, p 440.

13. Gowers WR. A manual of diseases of the nervous system, Vol II: Diseases of the brain and cranial nerves. General and functional diseases of the nervous system. Ed 2 (1893). Darien, Conn: Hafner Publ Co 1970, p 86.

14. Hawthome $\mathrm{CO}$. On the occurrence of a bi-lateral extensor response in states of unconsciousness. Practitioner 1914, 93: 330-333.

15. King LS. Signs and symptoms. JAMA 1968, 206: 1063-1065.

16. Littré E. Dictionaire de médecine, de chirurgie, de pharmacie et des sciences qui s'y rapportent. Ed 21. Paris: J.-B Baillière et Fils, 1908.

17. Louis ED, King D, Sacco RL, Mohr JP. Upper motor neuron signs: frequency and temporal development in patients with acute motor strokes. Ann Neurol 1993, 34: 291.

18. Noïca $\mathrm{D}$, Sakelaru. Le réflexe plantaire et le phénomène des orteils (signe de Babinski) au point de vue physiologique et physio-pathologique. Sem Méd 1906, 51: 601-602.

19. Oldendorf WH. The quest for an image of the brain: a brief historical and technical review of brain imaging techniques. Neurology 1978, 28:517-533.

20. Oliveira-Souza R. Motor hemiplegia and the cerebral organization of movement in man: I. The "pyramidal concept" - a restatement. Arq Neuropsiquiatr 1989, 47: 8-15.

21. Remak E. Zur Localisation der spinalen Hautreflexe der Unterextremitäten. Neurologisches Centralblatt 1893, 12: 506-512.

22. Sawyer RN, Hanna JP, Ruff RL, Leigh RJ. Asymmetry of forearm rolling as a sign of unilateral cerebral dysfunction. Neurology 1993, 43:1596-1598.

23. Wartenberg R. The Babinski reflex after fifty years. JAMA 1947, 135:763-767.

24. Yakovlev PI, Farrell MI. Influence of locomotion on the plantar reflex in normal and in physically and mentally inferior persons: theoretical and practical implications. Arch Neurol Psychiat 1941, 46:322-330. 